\title{
Mechanismen der Hautentzündung bei der Kontaktdermatitis
}

\author{
Mechanisms of Skin Inflammation in Contact Dermatitis
}

Autor

Institut

\author{
S. F. Martin
}

Forschergruppe Allergologie, Hautklinik, Universitätsklinikum Freiburg
Bibliografie

Dol http://dx.doi.org/

10.1055/s-0042-103772

Online-Publikation: 29.3.2016

Akt Dermatol 2016; 42: 344-348

(c) Georg Thieme Verlag KG

Stuttgart · New York

ISSN 0340-2541

Korrespondenzadresse

Prof. Dr. rer. nat.

Stefan F. Martin

Forschergruppe Allergologie Hautklinik

Universitätsklinikum Freiburg

Hauptstraße 7

79104 Freiburg

stefan.martin@uniklinik-freiburg. de

\section{Zusammenfassung \\ $\nabla$}

Viele Chemikalien können eine allergische Kontaktdermatitis verursachen. Diese wird von T-Zellen vermittelt, die Hautzellen schädigen. Voraussetzung für die Aktivierung und Differenzierung kontaktallergenspezifischer T-Zellen ist die Aktivierung des angeborenen Immunsystems. Die daraus resultierende Hautentzündung ähnelt mechanistisch der angeborenen Immunantwort gegen Bakterien und Viren. Diese Übersicht beschreibt die Orchestrierung der Hautentzündung bei der Kontaktallergie auf molekularer und zellulärer Ebene.

\section{Irritative und allergische Kontakt- dermatitis $\nabla$}

Die Kontaktdermatitis ist eine entzündliche Hauterkrankung, die meist durch niedermolekulare Chemikalien ausgelöst wird. Sie hat große sozioökonomische Auswirkungen, da sie häufig berufsbedingt auftritt $[1,2]$. Chemikalien, die Kontaktdermatitis verursachen, werden in Irritanzien und Kontaktallergene unterteilt. Irritanzien verursachen irritative Kontaktdermatitis, eine Ekzemreaktion, die ohne Beteiligung des sog. adaptiven Immunsystems, also ohne T- und B-Zellbeteiligung, schon bei Erstkontakt entsteht. Kontaktallergene, von denen bisher über 4000 bekannt sind, aktivieren kontaktallergenspezifische T-Zellen, die erst bei wiederholtem Kontakt zum klinischen Bild führen [3]. Die häufigsten Kontaktallergene werden regelmäßig erfasst [4]. Meist sind Kontaktallergene Duftstoffe oder Konservierungsmittel.

Kontaktallergene sind Haptene (Halbantigene), die erst nach Proteinbindung für das Immunsystem erkennbar sind. Die Proteinreaktivität ist dabei für ihre Funktion essentiell [5]. Manche Chemikalien werden erst nach oxidativer (Prä-Haptene) bzw. metabolischer Umwandlung (Pro-Hap- tene) zu proteinreaktiven Haptenen. Kontaktallergene dringen in die Haut ein und verursachen dort die Aktivierung von Hautzellen, Gewebestress und Gewebeschädigung und letztlich Hautentzündung [6]. Die molekularen und zellulären Prozesse, die von solchen Chemikalien aktiviert werden, sind bisher nur teilweise verstanden und nur für wenige Chemikalien im Detail untersucht. Der Erstkontakt, die Sensibilisierung, ist klinisch unauffällig, aber immunologisch entscheidend für die Entstehung der Kontaktallergie. Hier wird das sog. angeborene Immunsystem aktiviert und eine Hautentzündung ausgelöst, Voraussetzung für die Entstehung einer T-Zellantwort [7].

Die Stärke der Reaktion und ihre Dauer werden durch verschiedene Mechanismen reguliert. Sehr wichtig sind hier regulatorische T-Zellen (Treg), die durch Sekretion von Zytokinen wie IL-10, aber auch durch die Bildung von Adenosin sowohl auf antigenpräsentierende Zellen als auch Effektor-T-Zellen immunsuppressiv wirken $[8,9]$. Auch für invariante NKT-Zellen und regulatorische B-Zellen wurde eine Rolle in der Immunregulation der Kontaktallergie gezeigt $[10,11]$.

\section{Molekulare Mechanismen der Aktivie- rung des angeborenen Immunsystems durch Kontaktallergene $\nabla$}

Entzündung wird bei Infektionen durch die Aktivierung des angeborenen Immunsystems ausgelöst. Rezeptoren dieses Systems dienen als Sensoren für sog. danger signals [12]. Dies können Moleküle der Pathogene sein, so z. B. Lipopolysaccharid (LPS, Endotoxin) aus der Zellwand gram-negativer Bakterien, Nukleinsäuren oder Toxine. Diese Strukturen bezeichnet man als pathogen-associated molecular patterns (PAMPs) und die entsprechenden Rezeptoren als pattern recognition receptors (PRRs) [13]. Beispiele für PRRs sind die membranständigen Toll-like-Rezeptoren (TLR) wie 
TLR4, der LPS erkennt, oder die zytosolischen NOD-like-Rezeptoren (NLR) wie NLRP3, Bestandteil des NLRP3-Inflammasoms. Andere danger signals wirken über andere Rezeptoren oder auch unabhängig von Rezeptoren. Beispiele für körpereigene danger signals, die z. B. bei zellulärem Stress entstehen, sind extrazelluläres ATP und reaktive Sauerstoffspezies (reactive oxygen species, ROS).

Verschiedene PRR-Systeme sind meist funktionell gekoppelt. So führt die Aktivierung der TLRs über die Aktivierung des Transkriptionsfaktors NF-KB u.a. zur Synthese von unreifen Pro-Formen der Zytokine IL-1 $\beta$ und IL-18, wichtigen Entzündungsmediatoren. Die reifen, bioaktiven und sezernierten Zytokine entstehen dann durch Spaltung durch die Caspase-1, die durch das NLRP3-Inflammasom aktiviert wird. An der Aktivierung von NF-KB und des NLRP3-Inflammasoms sind auch ROS beteiligt.

Wir vermuteten, dass PRRs auch bei der Kontaktallergie eine Rolle spielen. Wir und andere zeigten dann, dass Mäuse, denen die TLR2 und TLR4, die normalerweise bakterielle Zellwandbestandteile erkennen, bzw. Interleukin-12-Rezeptor-beta-2 (IL-12Rß2) und einer dieser TLRs oder NLRP3 fehlen, praktisch resistent gegen Kontaktallergie sind. Dabei war die Funktionalität dieser PRRs in dendritischen Zellen (DCs) essentiell $[14,15]$. Bei der Frage, wie Kontaktallergene Entzündung auslösen, wurde vermutet, dass durch Schädigung der Hautbarriere möglicherweise PAMPs aus der Hautflora eindringen könnten. Jedoch ergaben unsere Studien im Mausmodell der Kontaktallergie, dem contact hypersensitivity (CHS)-Modell [16], dass Kontaktallergie in keimfreien Mäusen normal entsteht. Es ist bekannt, dass PRRs auch körpereigene Strukturen erkennen können, die dann als damage-associated molecular patterns (DAMPs) bezeichnet werden [17]. Das können Bestandteile der extrazellulären Matrix oder zelluläre Proteine wie HMGB1 oder auch Nukleinsäuren sein, die z. B. bei zellulärem Stress und Zellschädigung freigesetzt werden. Es stellte sich heraus, dass bei der CHS die Hyaluronsäure, ein Bestandteil der extrazellulären Matrix, oxidativ und enzymatisch fragmentiert wird [18]. Fragmente der Hyaluronsäure waren zuvor als Aktivatoren von TLR2 und TLR4 bei Lungenentzündung im Mausmodell beschrieben worden $[10,20]$. Interessanterweise verhinderte die Inhibition der Hyaluronidase im Mausmodell die Sensibilisierung [18].

Nickel- und Kobaltionen wirken direkt wie PAMPs. Diese binden an konservierte Histidine im humanen TLR4 und lösen eine Dimerisierung und Signalleitung aus. LPS ist hier nicht nötig [21, 22]. Dem TLR4 der Maus fehlen diese Histidine. Er kann daher diese Metallionen nicht binden. In der Maus kann auch durch Nickel alleine keine Kontaktallergie ausgelöst werden. Dazu muss zusätzlich ein danger signal wie LPS gegeben werden. Auch bei der Nickel-Kontaktdermatitis spielt das NLRP3-Inflammasom eine Rolle. Hier erfolgt die Aktivierung über mitochondriale ROS und Kationenströme [23].

Kontaktallergene können also entweder direkt PPRs aktivieren und Signalleitung auslösen oder indirekt, indem sie die Freisetzung oder Bildung von DAMPs auslösen.

\section{Zelluläre Mechanismen der Aktivierung des ange- borenen Immunsystems durch Kontaktallergene}

$\nabla$

Ein frühes Ereignis nach Hautkontakt mit Kontaktallergenen ist die Aktivierung von Mastzellen in der Dermis [24]. Obwohl die molekularen Mechanismen der Aktivierung nicht aufgeklärt sind, kommt es hier zur Ausschüttung von Histamin, einer erhöh- ten Permeabilität der Blutgefäße und früher Ödembildung. Als Konsequenz wird die Infiltration von neutrophilen Granulozyten gefördert, die in Abwesenheit von Mastzellen, wie im CHS-Mausmodell gezeigt, stark verringert ist. In diesem Modell ist auch die Ohrschwellungsreaktion in Mastzell-defizienten Mäusen und Mäusen, bei denen die Mastzellen vor Sensibilisierung depletiert wurden, stark vermindert [24]. Mastzellen haben nach diesen Ergebnissen eine entzündungsfördernde Wirkung bei der Kontaktallergie. Die Förderung der Infiltration von Neutrophilen ist eine wichtige Funktion der Mastzellen. Neue Untersuchungen haben gezeigt, dass die Neutrophilen ebenfalls eine essentielle entzündungsfördernde Wirkung bei der Kontaktallergie haben [25]. Im CHS-Modell zeigte sich eine Verhinderung der Kontaktallergie in Neutrophilen-defizienten Mäusen und in Mäusen, bei denen die Neutrophilen entweder vor Sensibilisierung oder vor Auslösung der Kontaktallergie entfernt wurden. Dies korrelierte mit der deutlichen Reduktion von MMP9-Aktivität, von Myeloperoxidase-abhängigen ROS und von Zytokinen, die für die CHS wichtig sind. Eine weitere Konsequenz war die Reduktion der Auswanderung von DCs aus der Haut in die drainierenden Lymphknoten, die ja Voraussetzung für die Aktivierung kontaktallergenspezifischer T-Zellen ist. In der Tat fand diese T-Zellaktivierung nicht statt. Interessanterweise waren Neutrophile auch für die Rekrutierung solcher T-Zellen in die Haut notwendig, da diese bei Depletion erst vor Auslösung der Kontaktallergie offensichtlich ausblieb. Neuere Daten in einem Mausmodell der Influenzainfektion zeigen, dass Neutrophile im Lungengewebe quasi eine Spur von Chemokinen im Gewebe legen, die die gezielte Rekrutierung von CD8+-T-Zellen ermöglicht [26]. Auch die Mechanismen der Neutrophilenaktivierung durch Kontaktallergene sind noch nicht aufgeklärt.

Diese Studien machen deutlich, dass es sich bei kontaktallergeninduzierter Entzündung in der Haut um ein komplexes Geschehen handelt, das sorgfältig orchestriert ist. Einen kleinen Ausschnitt aus dieser Orchestrierung zeigt auf zellulärer Ebene das essentielle Zusammenspiel von Mastzellen, Neutrophilen und DCs in der Haut. Auf molekularer Ebene wird die Aktivierung der DCs durch das Zusammenspiel von TLRs und NLRP3-Inflammason illustriert. Hierbei spielen ROS und ATP als endogene danger signals eine wichtige Rolle. Diese Erkenntnisse können jetzt zur Entwicklung kausaler, anti-entzündlicher Therapieansätze verwendet werden. Im CHS-Modell wurde das bereits erfolgreich demonstriert $[27,28]$. Inhibitoren der Hyaluronidase, Antioxidantien, Antagonisten des ATP-Rezeptors P2X7 R oder des IL-1-Rezeptors zeigten alle eine Inhibition der Sensibilisierung und z.T. auch der Auslösung der CHS.

Die enge funktionelle Kopplung der PRRs und der danger signals sowie der verschiedenen Zelltypen des angeborenen Immunsystems in der Orchestrierung der angeborenen Immunantwort gegen Kontaktallergene ermöglicht theoretisch eine therapeutische Intervention durch das Targeting nur eines Mechanismus, wie im CHS-Modell gezeigt.

\section{Adverse Arzneimittelreaktionen}

$\nabla$

Die Auslösung adverser Reaktionen gegen manche Arzneimittelwirkstoffe läuft ähnlich ab wie die Entstehung der Hautentzündung bei der Kontaktallergie. Bestes Beispiel hierfür ist Acetaminophen (APAP, Paracetamol). APAP kann bei langer hoher Dosierung zu Leberschäden führen. Dabei spielt das angeborene Immunsystem eine wichtige Rolle. APAP ist ein Pro-Hapten, das in 
der Leber metabolisch zum toxischen Metaboliten $N$-acetyl- $p$ benzochinon-imin (NAPQI) umgewandelt wird. Dieser kann Leberzellen schädigen, was zur Freisetzung von danger signals führt. Dabei dient dann körpereigene DNA als DAMP und aktiviert TLR9 auf sinusoidalen Leberendothelzellen (SLEC). Es kommt zur Bildung pro-inflammatorischer Zytokine, u.a. von pro-IL-1 $\beta$. Durch ATP und P2X7R sowie ROS wird das NLRP3-Inflammasom aktiviert und reifes IL-1 $\beta$ gebildet $[28,29]$. Die mechanistische Ähnlichkeit dieser adversen Arzneimittelreaktion und der angeborenen Immunantwort gegen Kontaktallergene legt nahe, dass auch hier anti-entzündliche Ansätze, wie im CHS-Modell gezeigt [30], wirksam sein könnten.

\section{Heterologe Stimulation des angeborenen Immunsystems}

Nickel kann nicht an den Maus-TLR4 binden und löst keine CHS aus. Allerdings führt die Kombination von Nickel und LPS zu Sensibilisierung und CHS [31]. Kontaktallergen-resistente Mäuse, denen z.B. TLR2 und TLR4 fehlen, können durch Kombination des Kontaktallergens 2,4,6,-Trinitrochlorbenzol (TNCB) mit einem Liganden für TLR9 suszeptibel gemacht werden und entwickeln dann CHS [14]. Die Kombination von Nickel mit SDS erhöht die Patch-Test-Reaktion [32]. Kombination von schwachen Kontaktallergenen [33] oder von Kontaktallergenen und Irritanzien [34] führt zur Augmentation der Kontaktallergie. Diese Befunde sind alle durch eine wichtige generelle Eigenschaft der angeborenen Immunantwort erklärbar, die hier am Beispiel der Kontaktallergie erläutert wird. Die T-Zellantwort ist kontaktallergen-spezifisch, d.h. der T-Zellrezeptor erkennt das Kontaktallergen direkt im Kontext von MHC-Molekülen [3]. Die angeborene Immunantwort, die Voraussetzung für die T-Zellantwort ist und letztlich zur Aktivierung von DCs und deren Wanderung aus der Haut in die Lymphknoten und dort zur T-Zellaktivierung führt, ist nicht notwendigerweise kontaktallergenspezifisch. Das heißt konkret, dass die Stimuli, die zur DC-Aktivierung führen, nicht alleine oder gar nicht von dem Kontaktallergen kommen müssen, gegen das sich die T-Zellantwort richtet. Während starke Kontaktallergene eine ausreichend starke Aktivierung des angeborenen Immunsystems erzielen (autologe Stimulation), ist das bei schwachen Kontaktallergenen wie z. B. vielen Duftstoffen nicht der Fall. Hier kann allerdings die Kombination mehrerer schwacher Kontaktallergene oder auch schwacher Kontaktallergene mit Irritanzien zu additiven oder synergistischen Effekten führen, die dann eine Sensibilisierung durch ein schwaches Kontaktallergen ermöglichen. Solche Kombinationen liegen in vielen Produkten wie Kosmetika oder Arbeitsstoffen vor, und die heterologe Stimulation führt dann zur Augmentation. Im Extremfall fehlt die autologe Stimulation durch ein Kontaktallergen ganz, wie im Fall von Nickel in der Maus oder den TLR2/4-defizienten Mäusen. Hier kann die heterologe Stimulation experimentell durch LPS bzw. einen TLR9-Liganden sogar zum Verlust einer Resistenz führen. Solche heterologen Stimuli können also durch andere Kontaktallergene, Irritanzien, danger signals, DAMPs oder PAMPs gegeben werden. Konsequenzen sind die Verstärkung einer sonst zu schwachen Aktivierung des angeborenen Immunsystems oder der Ersatz von fehlender Stimulation. So könnte z.B. eine Infektion ein Triggerfaktor sein, der Sensibilisierung ermöglicht, wie das die Kombination von LPS oder CpG mit einem Kontaktallergen impliziert [35].

\section{Fazit}

$\nabla$

Die Grundlagenforschung der letzten Jahre hat aufgezeigt, dass das Immunsystem auf Kontaktallergene wie auf eine Infektion reagiert. Die Mechanismen der Entzündung und die T-Zellantworten sind denen bei einer Infektion sehr ähnlich. Lediglich die Art und Weise, wie diese durch Chemikalien aktiviert werden, ist sehr speziell. Grundvoraussetzung für die Aktivität von Chemikalien ist ihre Proteinreaktivität, d. h. die Fähigkeit organischer Chemikalien kovalente Bindungen bzw. bei anorganischen Chemikalien wie Metallionen Komplexbindungen einzugehen. Bisher sind nur wenige Proteine bekannt, deren Modifikation funktionelle Konsequenzen hat. Das sind v. a. der humane TLR4, der durch Bindung von Nickel- oder Kobaltionen aktiviert wird, und Keap1, ein zytosolisches Sensorprotein für oxidativen und chemischen Stress, dessen Aktivierung durch Kontaktallergene an der Modulation der Entzündungsstärke beteiligt ist [36]. Eine wichtige Aufgabe der nächsten Jahre ist die Identifikation weiterer Zielproteine.

Das Konzept der heterologen Stimulation des angeborenen Immunsystems hat viele Implikationen und kann eine Reihe klinischer Befunde erklären. Entzündungsprozesse können dadurch verstärkt oder überhaupt erst ermöglicht werden.

Das Verständnis grundlegender immunologischer Mechanismen der Entstehung von Kontaktallergie kann sowohl für die Entwicklung neuer therapeutischer Ansätze als auch für die Entwicklung von In-vitro-Alternativen zum Tierversuch für die Kontaktallergenidentifikation in der Immuntoxikologie genutzt werden $[37,38]$. Der Einsatz globaler Technologien wie genomisches oder proteomisches Profiling von Zelllinien und Haut [39-44] führt zur Identifikation neuer Signalkaskaden, deren pathogenetische Relevanz jetzt überprüft werden kann. Man versucht hier auch charakteristische Gen- oder Proteinprofile zu identifizieren, die spezifisch für Kontaktallergene bzw. Irritanzien sind, spezifisch für bestimmte Kontaktallergene oder Klassen von Kontaktallergenen oder auch für bestimmte Ekzemformen $[45,46]$.

Darüber hinaus ist das Ziel dieser Arbeiten die Identifikation von Biomarkern, die zur Diagnostik der Kontaktallergie, aber auch anderer Ekzemtypen dienen können. Dies könnte längerfristig den Patch-Test ersetzen und z.B. eine klare Differenzierung von Kontaktekzem, atopischem und psoriatischem Ekzem durch moderne molekulare Diagnostik erlauben.

\section{Abkürzungen \\ $\nabla$ \\ ACD Allergische Kontaktdermatitis \\ CHS Kontakthypersensitivität \\ DAMP damage-associated molecular pattern \\ DC dendritische Zelle \\ HA Hyaluronsäure \\ ICD Irritative Kontaktdermatitis (ICD) \\ NLR NOD-like-Rezeptor \\ PAMP pathogen-associated molecular pattern \\ ROS reaktive Sauerstoffspezies \\ TLR Toll-like-Rezeptor}

\section{Interessenkonflikt}

$\nabla$

Der Autor gibt an, dass kein Interessenkonflikt besteht. 


\section{Mechanisms of Skin Inflammation in Contact Dermatitis \\ $\nabla$}

Many chemicals can cause allergic contact dermatitis. This skin disease is mediated by $\mathrm{T}$ cells which damage skin cells. A prerequisite for the activation and differentiation of contact allergen specific T cells is the activation of the innate immune system. The resulting skin inflammation mechanistically resembles the innate immune response to bacteria and viruses. This overview describes the orchestration of skin inflammation in allergic contact dermatitis at the molecular and cellular level.

\section{Literatur}

1 Peiser M, Tralau T, Heidler J et al. Allergic contact dermatitis: epidemiology, molecular mechanisms, in vitro methods and regulatory aspects. Current knowledge assembled at an international workshop at BfR, Germany. Cell Mol Life Sci 2012; 69: 763 - 781. DOI: 10.1007/s00018011-0846-8

2 Wiszniewska M, Walusiak-Skorupa J. Recent Trends in Occupational Contact Dermatitis. Current allergy and asthma reports 2015; 15: 43. DOI: $10.1007 / \mathrm{s} 11882-015-0543-\mathrm{z}$

3 Martin SF, Esser PR, Schmucker S et al. T-cell recognition of chemicals, protein allergens and drugs: towards the development of in vitro assays. Cell Mol Life Sci 2010; 67: 4171 -4184. DOI: 10.1007/s00018010-0495-3

4 Mahler V, Geier J, Schnuch A. Current trends in patch testing - new data from the German Contact Dermatitis Research Group (DKG) and the Information Network of Departments of Dermatology (IVDK). Journal der Deutschen Dermatologischen Gesellschaft $=$ Journal of the German Society of Dermatology: JDDG 2014; 12: 583-592. DOI: 10.1111/ ddg. 12371

5 Martin SF. Contact dermatitis: from pathomechanisms to immunotoxicology. Exp Dermatol 2012; 21: 382-389. DOI: 10.1111/j.16000625.2012.01471.x

6 Martin SF, Esser PR. Chemical allergen-induced skin cell activation. In: Corsini E, Van Loveren H, eds. Molecular Immunotoxicology. 1 edn. Weinheim: Wiley; 2014: 91 -116

7 Iwasaki A, Medzhitov R. Control of adaptive immunity by the innate immune system. Nat Immunol 2015; 16: 343 -353. DOI: 10.1038/ ni.3123

8 Kimber I, Travis MA, Martin SF et al. Immunoregulation of skin sensitization and regulatory T cells. Contact Dermatitis 2012; 67: 179-183. DOI: $10.1111 / j .1600-0536.2012 .02148 . x$

9 Ring S, Oliver SJ, Cronstein BN et al. CD4+CD25+ regulatory T cells suppress contact hypersensitivity reactions through a CD39, adenosinedependent mechanism. J Allergy Clin Immunol 2009; 123: 1287 1296, e1282. DOI: $10.1016 /$ j.jaci.2009.03.022

10 Goubier A, Vocanson M, Macari C et al. Invariant NKT cells suppress CD8 $(+)$ T-cell-mediated allergic contact dermatitis independently of regulatory CD4(+) T cells. J Invest Dermatol 2013; 133: 980-987. DOI: $10.1038 /$ jid.2012.404

11 Nakashima $H$, Hamaguchi $Y$, Watanabe $R$ et al. CD22 expression mediates the regulatory functions of peritoneal B-1a cells during the remission phase of contact hypersensitivity reactions. J Immunol 2010; 184 : 4637-4645. DOI: 10.4049/jimmunol.0901719

12 Matzinger $P$. The danger model: a renewed sense of self. Science 2002; 296: 301 - 305. DOI: 10.1126/science. 1071059

13 Janeway CA Jr. Approaching the asymptote? Evolution and revolution in immunology. Cold Spring Harb Symp Quant Biol 1989; 54: 1 - 13

14 Martin SF, Dudda JC, Bachtanian E et al. Toll-like receptor and IL-12 signaling control susceptibility to contact hypersensitivity. J Exp Med 2008; 205: 2151-2162. DOI: 10.1084/jem.20070509

15 Sutterwala FS, Ogura Y, Szczepanik $M$ et al. Critical role for NALP3/CIAS1/Cryopyrin in innate and adaptive immunity through its regulation of caspase-1. Immunity 2006; 24: $317-327$. DOI: 10.1016/j.immuni.2006.02.004
16 Martin SF. Induction of contact hypersensitivity in the mouse model. Methods in molecular biology 2013; 961: 325-335. DOI: 10.1007/ 978-1-62703-227-8_21

17 Seong SY, Matzinger P. Hydrophobicity: an ancient damage-associated molecular pattern that initiates innate immune responses. Nat Rev Immunol 2004; 4: 469-478. DOI: 10.1038/nri1372

18 Esser PR, Wolfle U, Durr C et al. Contact sensitizers induce skin inflammation via ROS production and hyaluronic acid degradation. PLoS One 2012; 7: e41340. DOI: 10.1371/journal.pone.0041340

19 Jiang D, Liang J, Fan J et al. Regulation of lung injury and repair by Tolllike receptors and hyaluronan. Nat Med 2005; 11: 1173-1179. DOI: $10.1038 / \mathrm{nm} 1315$

20 Scheibner KA, Lutz MA, Boodoo $S$ et al. Hyaluronan fragments act as an endogenous danger signal by engaging TLR2. J Immunol 2006; 177: 1272 - 1281. DOI: $177 / 2 / 1272$ [pii]

21 Schmidt M, Raghavan B, Muller V et al. Crucial role for human Toll-like receptor 4 in the development of contact allergy to nickel. Nat Immunol 2010; 11: 814-819. DOI: 10.1038/ni.1919

22 Raghavan B, Martin SF, Esser PR et al. Metal allergens nickel and cobalt facilitate TLR4 homodimerization independently of MD2. EMBO reports 2012; 13: 1109-1115. DOI: 10.1038/embor.2012.155

$23 \mathrm{Li}$, Zhong $F$. Nickel induces interleukin-1 beta secretion via the NLRP3ASC-caspase-1 pathway. Inflammation 2014; 37: 457-466. DOI: 10.1007/s10753-013-9759-z

24 Dudeck A, Dudeck J, Scholten J et al. Mast Cells Are Key Promoters of Contact Allergy that Mediate the Adjuvant Effects of Haptens. Immunity 2011; 34: 973-984. DOI: 10.1016/j.immuni.2011.03.028

25 Weber FC, Nemeth T, Csepregi JZ et al. Neutrophils are required for both the sensitization and elicitation phase of contact hypersensitivity. J Exp Med 2015; 212: 15 - 22. DOI: 10.1084/jem.20130062

26 Lim K, Hyun YM, Lambert-Emo $K$ et al. Neutrophil trails guide influenza-specific CD8(+) T cells in the airways. Science 2015; 349: aaa4352. DOI: $10.1126 /$ science.aaa4352

27 Martin SF, Esser PR, Weber FC et al. Mechanisms of chemical-induced innate immunity in allergic contact dermatitis. Allergy 2011; 66: 1152 - 1163. DOI: 10.1111/j.1398-9995.2011.02652.x

28 Imaeda $A B$, Watanabe A, Sohail MA et al. Acetaminophen-induced hepatotoxicity in mice is dependent on Tlr9 and the Nalp3 inflammasome. J Clin Invest 2009; 119: 305 - 314. DOI: 10.1172/JCI35958

29 Hoque R, Sohail MA, Salhanick S et al. P2X7 receptor-mediated purinergic signaling promotes liver injury in acetaminophen hepatotoxicity in mice. Am J Physiol Gastrointest Liver Physiol 2012; 302: G1171 - 1179. DOI: 10.1152/ajpgi.00352.2011

30 Martin SF. Novel concepts of immune responses to chemicals in allergic contact dermatitis. Allergo journal international 2016; 25: 17-21. DOI: $10.1007 / \mathrm{s} 40629-016-0090-8$

31 Kinbara M, Sato N, Kuroishi $T$ et al. Allergy-inducing nickel concentration is lowered by lipopolysaccharide at both the sensitization and elicitation steps in a murine model. Br J Dermatol 2011; 164: 356-362. DOI: $10.1111 / \mathrm{j} .1365-2133.2010 .10016 . \mathrm{x}$

32 Agner T, Johansen JD, Overgaard $L$ et al. Combined effects of irritants and allergens. Synergistic effects of nickel and sodium lauryl sulfate in nickel- sensitized individuals. Contact Dermatitis 2002; 47: 21-26

33 Bonefeld CM, Nielsen MM, Rubin IM et al. Enhanced sensitization and elicitation responses caused by mixtures of common fragrance allergens. Contact Dermatitis 2011; 65: 336-342. DOI: 10.1111/j.16000536.2011.01945.x

34 Pedersen LK, Johansen JD, Held E et al. Augmentation of skin response by exposure to a combination of allergens and irritants - a review. Contact Dermatitis 2004; 50: 265-273. DOI: 10.1111/j.01051873.2004.00342.x

35 Martin SF. Adaptation in the innate immune system and heterologous innate immunity. Cell Mol Life Sci 2014; 71: 4115-4130. DOI: 10.1007/s00018-014-1676-2

36 El AliZ, Gerbeix C, Hemon P et al. Allergic skin inflammation induced by chemical sensitizers is controlled by the transcription factor Nrf2. Toxicol Sci 2013; 134: 39-48. DOI: 10.1093/toxsci/kft084

37 Reisinger $K$, Hoffmann $S$, Alepee $N$ et al. Systematic evaluation of nonanimal test methods for skin sensitisation safety assessment. Toxicol In Vitro 2015; 29: 259-270. DOI: 10.1016/j.tiv.2014.10.018

38 Urbisch $D$, Mehling A, Guth $K$ et al. Assessing skin sensitization hazard in mice and men using non-animal test methods. Regul Toxicol Pharmacol 2015; 71: 337-351. DOI: 10.1016/j.yrtph.2014.12.008 
39 Johansson H, Albrekt AS, Borrebaeck CA et al. The GARD assay for assessment of chemical skin sensitizers. Toxicol In Vitro 2013; 27: 1163 1169. DOI: 10.1016/j.tiv.2012.05.019

40 Lambrechts $N$, Nelissen I, Van Tendeloo Vet al. Functionality and specificity of gene markers for skin sensitization in dendritic cells. Toxicol Lett 2011; 203: 106 -110. DOI: 10.1016/j.toxlet.2011.02.015

41 Cottrez F, Boitel E, Auriault C et al. Genes specifically modulated in sensitized skins allow the detection of sensitizers in a reconstructed human skin model. Development of the SENS-IS assay. Toxicol In Vitro 2015; 29: 787 -802. DOI: 10.1016/j.tiv.2015.02.012

42 van der Veen JW, Pronk TE, van Loveren $H$ et al. Applicability of a keratinocyte gene signature to predict skin sensitizing potential. Toxicol In Vitro 2013; 27: 314-322. DOI: 10.1016/j.tiv.2012.08.023
43 van der Veen JW, Hodemaekers $H$, Reus AA et al. Human relevance of an in vitro gene signature in $\mathrm{HaCaT}$ for skin sensitization. Toxicol In Vitro 2014; 29: 81 -84. DOI: 10.1016/j.tiv.2014.08.010

44 Vandebriel RJ, Pennings JL, Baken KA et al. Keratinocyte gene expression profiles discriminate sensitizing and irritating compounds. Toxicol Sci 2010; 117: 81 -89. DOI: 10.1093/toxsci/kfq182

45 Dhingra N, Shemer A, Correa da Rosa J et al. Molecular profiling of contact dermatitis skin identifies allergen-dependent differences in immune response. J Allergy Clin Immunol 2014; 134: 362 -372. DOI: 10.1016/j.jaci.2014.03.009

46 Quaranta M, Knapp B, Garzorz N et al. Intraindividual genome expression analysis reveals a specific molecular signature of psoriasis and eczema. Sci Transl Med 2014; 6: 244ra290. DOI: 10.1126/scitranslmed.3008946 